\title{
Juventudes en América Latina y el Caribe en perspectiva: panorama de la situación, desafíos e intervenciones promisorias
}

\author{
Youth in Latin America and the Caribbean in perspective: \\ overview of the situation, challenges and promising interventions
}

José Roberto Luna Manzanero (https://orcid.org/0000-0002-8650-743X) ${ }^{1}$

\footnotetext{
${ }^{1}$ Technical Division, Sexual and Reproductive Health Branch, United Nations Population Fund (UNFPA). 605 Third Avenue, New York NY 10158 USA.

jluna@unfpa.org
}

\begin{abstract}
This article approaches, from a youth perspective, an overview of the situation of youth in Latin America and the Caribbean based on global and regional socio-economic and demographic indicators. It provides an intersectional analysis of the oppressions and challenges that young people face to achieve their full potential within a complex structure of power relations, inequality, exclusion, discrimination and violence. Based on the evidence, it highlights effective and promising interventions for guiding investment in youth through public policies, budgets and programmes at scale, as measures for redistributing power and resources that contribute to the fulfilment of their human rights, autonomy, emancipation and agency to participate in public affairs that affect them.

Key words Youth, Violence and health, Sexual and reproductive health and rights, Promising practices, Latin America and the Caribbean
\end{abstract}

Resumen Este artículo aborda, desde la perspectiva de juventud, un panorama de la situación de las juventudes en América Latina y el Caribe a partir de indicadores socioeconómicos y demográficos globales y regionales. Provee un análisis interseccional de las opresiones y desafíos que enfrentan las personas jóvenes para alcanzar su pleno desarrollo dentro de un entramado de relaciones de poder, desigualdad, exclusión, discriminación $y$ violencia. Con base en evidencias, destaca intervenciones eficaces y promisorias que orienten la inversión en juventud mediante políticas públicas, presupuestos y programas a escala, como medidas redistributivas del poder y los recursos que contribuyan al disfrute de sus derechos humanos, la autonomía, emancipación y agencia para participar en asuntos públicos que les afectan.

Palabras clave Juventudes, Violencia y salud, Derechos sexuales y derechos reproductivos, Prácticas promisorias, América Latina y el Caribe 


\section{Introducción}

El mundo es hoy el hogar de 1,800 millones de jóvenes, quienes representan una cuarta parte de la población global y constituyen una fuerza vital que se desarrolla en medio de cambios históricos y transformaciones políticas, sociales, económicas y ambientales que determinan su presente y futuro ${ }^{1}$.

Este artículo tiene como objetivos: a) ofrecer un análisis panorámico de la situación de las juventudes en América Latina y el Caribe (ALC); b) analizar interseccionalmente las opresiones y desafíos que enfrentan para su desarrollo; y c) destacar prácticas promisorias basadas en evidencia que orienten la inversión en políticas públicas a escala. La metodología utilizada partió de la revisión de literatura global y regional en torno a los principales indicadores socioeconómicos y demográficos desde la perspectiva de juventudes; el análisis interseccional de los desafíos identificados a partir de los datos y la realidad; y la revisión de evidencias, marcos normativos y estándares internacionales para identificar prácticas promisorias implementadas en la región que orienten inversiones públicas.

Las juventudes representan oportunidades para el desarrollo de los países y de la región de ALC, no obstante, el contexto sostenido de desigualdad estructural limita sus oportunidades de vida, por ello, a partir del análisis de la realidad, la evidencia y el marco de derechos humanos e igualdad de género; se proponen una serie de intervenciones promisorias que orienten la inversión en juventudes como una medida de justicia redistributiva que les permita generar agencia y condiciones para su pleno desarrollo, con dignidad y derechos humanos.

\section{Situación actual de las juventudes, un panorama de América Latina y el Caribe}

En ALC se estima que actualmente viven alrededor de 165 millones de personas jóvenes entre los 10 y los 24 años de edad, lo que significa que una de cada cuatro personas en la región es jo$v^{2}{ }^{2}$. Pese a los avances obtenidos en ALC en la reducción de la pobreza entre 2002-2014, a partir de 2015 los niveles de pobreza y especialmente de pobreza extrema aumentaron. En 2018, alrededor del 30,1\% de la población regional estaba bajo la línea de pobreza, mientras que un 10,7\% se encontraba bajo el umbral de la pobreza extrema. Aproximadamente 185 millones de personas se encontraban en situación de pobreza, de las cuales 66 millones estaban en situación de pobreza extrema. Entre 25 y 30 millones de personas en las Américas corren el riesgo de caer de nuevo en la pobreza, incluyendo a las juventudes quienes enfrentan las consecuencias de la desigualdad a través de la falta de oportunidades educativas, vocacionales y de empleo decente. En 2016, sólo el 59,5\% de las personas jóvenes entre 20-24 años habían completado la educación secundaria, mostrando además desigualdades socioeconómicas; el $83,5 \%$ del quinto quintil había completado este nivel educativo versus el $35,4 \%$ del primer quintil. Esta situación también se refleja en el acceso a la formación profesional y universitaria, y la estratificada calidad educativa; inaccesible para todas las personas jóvenes s $^{3,4}$.

Según datos censales disponibles en 2010, en ALC la población indígena era aproximadamente 42 millones de personas quienes representaban el $8 \%$ de la población total. Si bien la pobreza afecta a muchos sectores de la población de la región, ésta afecta desproporcionadamente a los pueblos indígenas. El 43\% de los hogares indígenas viven en pobreza y el $24 \%$ en pobreza extrema, limitando las oportunidades de vida y desarrollo de las personas, incluyendo a las juventudes ${ }^{5}$. Los pueblos afrodescendientes representan el $24 \%$ de la población, equivalente a 130 millones de personas quienes enfrentan desigualdades y brechas de equidad que impactan su pleno desarrollo ${ }^{6}$.

El desempleo juvenil, es otro factor estructural que marca las trayectorias de las juventudes en ALC donde la tasa de desocupación juvenil triplica la de la población adulta, uno de cada cinco jóvenes en la región busca trabajo y no lo encuentra. En 2019 las juventudes experimentaron impactos negativos en el campo laboral, la tasa de desocupación juvenil aumentó 0,3 puntos y afectó cerca del $20 \%$ de los jóvenes que buscan trabajo en la región ${ }^{7}$. Esto sumado a que uno de cada cinco jóvenes no estudia ni trabaja en la región de ALC representando más de 20 millones de personas entre 15 y 24 años, situación que afecta principalmente a las mujeres jóvenes $(66 \%)$ y está ligado a condiciones de pobreza, vulnerabilidad y exclusión, que en contextos como México y Centroamérica se vincula, además, a la situación de violencia y delincuencia ${ }^{8}$. El trabajo de cuidado afecta la capacidad de las mujeres, incluidas las jóvenes, de obtener empleos, en ALC la dedicación de las mujeres al trabajo doméstico y de cuidados no remunerado ronda entre un quinto y un tercio de su tiempo diario o semanal, mientras que los hombres dedican cerca del $10 \%$ y más del $43 \%$ de las mujeres declara que no busca 
un empleo remunerado porque debe atender tareas de cuidado, trabajo doméstico o por la prohibición de algún familiar'.

La violencia, la situación socioeconómica, la falta de empleo y oportunidades inducen la movilidad humana de las juventudes en ALC. La migración interna e internacional, incluida la migración intrarregional, adquiere características particulares según la subregión; las personas de México y Centroamérica suelen emigrar hacia Estados Unidos, mientras en Sudamérica la predominancia es la migración entre países. En Centroamérica la situación socioeconómica y la violencia fomentan la migración de mujeres, niñas, niños y adolescentes ${ }^{10}$ quienes incluso viajan no acompañados poniendo en riesgo su vida e integridad en el tránsito, donde pueden ser víctimas de una serie de violaciones de derechos, incluida la violencia sexual, la explotación y el tráfico de personas. La migración forzada afecta a países centroamericanos por violencia social y económica, a Colombia producto de la violencia emanada del conflicto armado interno y a Venezuela por la crisis económica y sociopolítica ${ }^{11,12}$.

Aunque la tasa de fecundidad total ha ido disminuyendo en ALC a lo largo de un poco más de tres décadas, la tasa de fecundidad en adolescentes solo ha disminuido ligeramente durante ese período y sigue siendo la segunda más alta del mundo, sólo superada por África Subsahariana. En 2019, la tasa de fecundidad en adolescentes se calculaba en 61 nacimientos por cada 1000 mujeres de 15 a 19 años, en comparación con una tasa de 42 en el resto del mundo ${ }^{13,14}$. Si bien la región tiene una tasa de prevalencia de $57 \%$ en el uso de anticonceptivos modernos en adolescentes, la proporción de nacimientos de madres adolescentes representa el 20\% siendo la proporción regional más alta del mundo ${ }^{15}$.

La primera relación sexual, el primer matrimonio y el primer nacimiento son algunos de los acontecimientos vitales que marcan las trayectorias de las personas jóvenes, en particular las de las mujeres. La edad mediana de la primera relación sexual, el primer matrimonio y el primer nacimiento han aumentado en el África subsahariana, al igual que el primer matrimonio y el primer nacimiento en Asia y África septentrional. En ALC, el momento del primer nacimiento no cambió significativamente y la edad mediana regional de la primera relación sexual disminuyó considerablemente ${ }^{16}$.

Mientras otras regiones han logrado avances en la reducción del matrimonio infantil -matrimonio o unión formal o informal en el que uno o ambos cónyuges son menores de 18 años-en ALC la prevalencia $(25 \%)$ es decir, una de cada cuatro mujeres jóvenes contrajo matrimonio por primera vez o mantenía una unión temprana antes de cumplir los 18 años, situación que no ha variado en los últimos 25 años. Los datos son más alarmantes en países cuya prevalencia supera el 30\%, tales como la República Dominicana, Nicaragua, Honduras y Belice. Asimismo, México, Brasil y Colombia se encuentran entre los países con la mayor incidencia de matrimonio infantil a nivel global. En términos de masculinidades, los países de ALC tienen unas de las tasas más elevadas de matrimonio infantil de varones en el mundo. De los 10 países que cuentan con datos disponibles, 9 se ubican por encima del promedio mundial ${ }^{17,18}$.

Los homicidios son la tercera causa de mortalidad para los hombres a nivel global. Según datos de 133 países, en países de ingresos bajos y medianos, las tasas estimadas de homicidio más elevadas se registran en la región de las Américas, con 28,5 homicidios por 100.000 habitantes, seguida por África con una tasa de 10,9 homicidios por 100.000 habitantes ${ }^{19}$. Un análisis realizado con base en la Encuesta Mundial de Salud Escolar demuestra que los hombres jóvenes tienen mayor probabilidad de involucrarse en actos violentos, como la agresión y peleas físicas, en comparación a sus compañeras mujeres. No obstante, la intimidación, no presenta especificidad por sexo ${ }^{20}$.

La violencia contra las mujeres afecta a una de cada tres mujeres globalmente, quienes han sido objeto de violencia física y/o sexual por parte de la pareja o de violencia sexual fuera de la pareja a lo largo de su vida, 38\% de las muertes de mujeres han sido ocasionadas por su pareja íntima y 7\% de las mujeres han sido víctimas de violencia sexual por alguien que no es su pareja ${ }^{21}$. Información oficial proporcionada al Observatorio de Igualdad de Género de ALC de la Comisión Económica para América Latina y el Caribe (CEPAL), en 2019 al menos 4,555 mujeres de 18 países fueron víctimas de femicidio, la máxima expresión de la violencia contra las mujeres y niñas ${ }^{22}$. La socialización de género inmersa en imaginarios sociales violentos y normas sociales de género inequitativas sostenidas por sistemas de poder patriarcales, hace que las juventudes en la región de ALC naturalicen la violencia machista. Un estudio realizado en 8 países con personas jóvenes de 15 a 25 años de ALC muestra que en el grupo de adolescentes de 15 a 19 años el 65\% de los hombres y $45 \%$ de las mujeres, creen que las mujeres se hacen las difíciles, dicen no, pero en realidad quieren decir sí, creencia que naturaliza 
la violencia sexual; $87 \%$ de las personas jóvenes creen que los hombres tienen mayor deseo sexual que las mujeres, $77 \%$ percibe como normal que los hombres tengan relaciones sexuales con otras personas y es mal visto si las mujeres lo hacen y; el acoso callejero tiene un 77\% de aceptación como algo natural ${ }^{23}$. Otra manifestación de la violencia basada en género (VBG), es la violencia contra las personas lesbianas, gais, bisexuales, trans e intersexuales (LGBTI) con base en su orientación sexual o identidad de género. Diversas organizaciones civiles en ALC señalan que la expectativa de vida de las mujeres trans en la región es de 30 a 35 años de edad y según información estadística recolectada por la Comisión Interamericana de Derechos Humanos (CIDH) 80\% de las personas trans asesinadas durante un período de 15 meses tenía 35 años de edad o menos ${ }^{24}$.

La violencia autoinfligida es otra realidad que viven las juventudes de ALC, según la Organización Mundial de la Salud (OMS) 2014, el suicidio es la cuarta causa de muerte entre las y los adolescentes de 10 a 19 años de edad en las Américas. Estimaciones realizadas con base en los datos de la Encuesta Mundial de Salud Escolar muestran que las jóvenes tienen mayores probabilidades que los jóvenes varones de manifestar comportamientos suicidas. La prevalencia de ideación suicida en varones oscilaba alrededor de $11 \%$ en Centroamérica y el Cono Sur, y $17 \%$ en el Caribe de habla inglesa, en mujeres oscilaba entre $19 \%$ en el Cono Sur y $26 \%$ en los países andinos. Los intentos reales de suicidio en varones oscilaban entre 9\% en Centroamérica y 17\% en el Caribe de habla inglesa, en mujeres oscilaba entre $17 \%$ en Centroamérica y $23 \%$ en los países andinos, respectivamente ${ }^{25}$.

Las personas jóvenes con discapacidad experimentan mayores riesgos de sufrir violencia. Globalmente, una de cada ocho personas adultas vive con una discapacidad, esto equivale a más de mil millones de personas. En ALC la proporción es similar, las personas con discapacidad representan alrededor del $13 \%$ de la población ${ }^{26}$. Las mujeres con discapacidad están expuestas a un mayor riesgo de sufrir violencia que los hombres con discapacidad y que las mujeres sin discapacidad, encontrándose en mayor desventaja que sus pares hombres, con menos oportunidades laborales que las hace vulnerables a vivir en condiciones de pobreza y subordinación, por lo tanto, con menos poder y recursos para romper círculos de violencia y discriminación ${ }^{27}$.

El panorama de la situación socioeconómica y demográfica de las juventudes en ALC, dista de reflejar sociedades de bienestar social que brinden garantías de derechos fundamentales en condiciones de igualdad, libres de discriminación y violencias.

\section{Desafíos que enfrentan las juventudes de América Latina y el Caribe}

América Latina y el Caribe, si bien no es la región más pobre del mundo, es la región más desigual ${ }^{28}$ esto estructura una serie de desafíos que se cruzan en los cuerpos y las vidas de millones de personas jóvenes y obstaculizan su pleno desarrollo y bienestar. Hace 25 años tres grandes hitos establecieron rutas globales para abordar estos desafíos: 1) Declaración y Programa de Acción de la Conferencia Mundial de Derechos Humanos, Viena 1993; 2) Programa de Acción de la Conferencia Internacional sobre Población y Desarrollo (CIPD) Cairo 1994; 3) Plataforma de Acción Mundial de la Cuarta Conferencia Mundial sobre la Mujer, Beijing 1995 $29-31$ estos marcos normativos establecieron un antes y un después en la comprensión de los derechos humanos de las mujeres, en particular, las relaciones entre los asuntos de población y el desarrollo, el reconocimiento de la sexualidad como un asunto de derechos y trazó una visión clara y visionaria respecto a la urgencia de avanzar en asuntos de igualdad de género.

El análisis del progreso a 25 años de la CIPD muestra que ha habido avances globales en la reducción de embarazos en adolescentes y el matrimonio infantil, aunque en ALC estos indicadores muestran brechas importantes que deben ser abordadas de forma sistémica por los gobiernos, la sociedad civil, la academia y los generadores de opinión pública. Se ha avanzado en la generación de evidencias, programas y políticas para responder a la salud sexual y reproductiva (SSR) de adolescentes y jóvenes, no obstante, alrededor del mundo siguen existiendo resistencias que impiden el avance en la provisión de un paquete integral de servicios de SSR para las personas jóvenes desde un marco que respete los derechos sexuales y reproductivos (DSR). Otros indicadores como la violencia por parte de la pareja han empeorado, generando un nuevo llamado a abordar con mayor sistematicidad, programas de masculinidades con base en evidencias y enfoques de género transformadores que involucren a hombres y niños en asuntos de igualdad entre los géneros ${ }^{32,33}$.

Los datos disponibles permiten hacer un análisis interseccional de los desafios que enfrentan las 
juventudes por condición de edad, género, clase, raza, orientación sexual e identidad de género y discapacidades, dentro de un sistema de relaciones desiguales de poder que determina accesos, oportunidades, discriminación y exclusión: (a) Adultismo: Juventudes experimentan discriminación, exclusión, abuso de poder y autoridad, menores oportunidades de empleo decente y niveles de ingresos, desinversión, negación del derecho a la participación, ejercicio de la sexualidad, autodeterminación, autonomía y emancipación; (b) Capacitismo: Juventudes con discapacidad enfrentan discriminación, exclusión, invisibilización, violencia, inaccesibilidad y desprotección social; (c) Clasismo: viven desigualdad, migración forzada, estratificación en el acceso y disfrute de derechos como la educación, la salud -sexual y reproductiva, mental, nutricional, etc.-, la seguridad, el empleo y la seguridad social; $(d)$ Heterosexismo: Juventudes LGBTI viven discriminación por orientación sexual e identidad de género, violencia, acoso, exclusión, violencia simbólica, vulneración de derechos, falta de acceso a servicios, suicidio, crímenes de odio y menor expectativa de vida; (e) Racismo: Juventudes indígenas y afrodescendientes enfrentan discriminación, racismo, desigualdad, despojo de territorios y recursos naturales, exclusión en salud, educación, empleo e ingresos de forma sistémica e inaccesibilidad a servicios interculturales; migrantes, desplazados internos y refugiados sufren discriminación y xenofobia; y $(f)$ Sexismo: Mujeres jóvenes sufren $\mathrm{VBG}$, acoso callejero, violencia sexual y de pareja, discriminación, embarazo en la adolescencia, matrimonio infantil, femicidios, suicidio, violencia epistémica y obstétrica. Hombres jóvenes sufren la violencia social, homicida y física derivado de mandatos de la masculinidad hegemónica.

Abordar los desafíos requiere transformar las estructuras, las relaciones y la distribución del poder y los recursos, y que las juventudes puedan ejercer ciudadanías con agencia que les permitan acceder mediante la participación, a espacios, redes y recursos que garanticen su bienestar y derechos humanos. Esto implicaría que accedan al: (a) Poder sobre: a través de políticas de redistribución de recursos y de reconocimiento, que incluye, además, el ejercicio de los DSR; (b) Poder para: mediante políticas sostenidas de acceso a conocimientos, habilidades y oportunidades para que las juventudes alcancen su pleno potencial; (c) Poder dentro: Atañe a la agencia personal ligada al sentido de identidad, autovalor y reconocimiento de los propios derechos para tomar las riendas de su historia personal, autonomía y emancipación; y (d) Poder con: al participar colectivamente para alcanzar sociedades más justas y equitativas, valorando la diversidad, y construyendo solidaridad intergeneracional ${ }^{34}$.

\section{Invertir en las juventudes en América Latina y el Caribe, intervenciones promisorias e instrumentos para la acción}

La evidencia global ha planteado que la inversión en la salud de las y los adolescentes genera un triple dividendo, es decir beneficios en el presente, en el futuro como personas adultas y hasta la siguiente generación. Este triple dividendo podría tener efectos positivos en la salud, el bienestar y en el desarrollo de capacidades para la vida productiva y para la crianza efectiva de las generaciones venideras. Por ello, las inversiones a gran escala en la adolescencia y juventud deberían considerarse como estrategias esenciales e intergeneracionales para la salud y el bienestar de todas las sociedades ${ }^{35}$. Dichas inversiones en juventud deberían estar orientadas por la evidencia de intervenciones eficaces o promisorias, y observar compromisos internacionales provenientes de instrumentos normativos como el Programa de Acción de la CIPD, la Plataforma de Acción de Beijing, los Objetivos de Desarrollo Sostenible, así como documentos técnicos como la Estrategia Mundial para la Salud de la Mujer, el Niño y el Adolescente (2016-2030), la Guía de Acción Acelerada para la Salud de Adolescentes (AA-HA! por sus siglas en inglés), la estrategia global de UNFPA Mi Vida, Mi Cuerpo, Mi Mundo y la Serie de Orientaciones sobre Prevención de la Violencia de OMS; además de instrumentos regionales como el Consenso de Montevideo sobre Población y Desarrollo y su Guía Operacional y la iniciativa 165 Millones de Razones que delinean rutas estratégicas a seguir para invertir en intervenciones a niveles de las políticas en la región de $\mathrm{ALC}^{36-38}$.

Las políticas públicas son instrumentos de carácter político-técnicos que dirigen las acciones de los gobiernos, en asuntos de interés público y que tendrán un impacto a escala en la vida de las poblaciones ${ }^{39}$ las cuales para ser operativas deben traducir la voluntad política en programas, estrategias, planes y presupuestos nacionales orientados a implementar intervenciones basadas en evidencia como medidas de redistribución destinadas a generar cambios sociales, económicos, políticos y culturales que garanticen los derechos y el bienestar de las juventudes. Entre las intervenciones eficaces y promisorias se destacan: 
- Programas de educación integral en sexualidad (EIS) este enfoque educativo basado en los derechos humanos y el género, cuando se aplica dentro o fuera de la escuela ha demostrado tener efectos positivos en retrasar el inicio de las relaciones sexuales, menor frecuencia en las relaciones sexuales, menor cantidad de parejas sexuales y comportamientos de riesgo, y mayor uso de condones y de anticonceptivos. Cuando se implementan con un enfoque fuerte en género y empoderamiento muestran mayor eficacia ${ }^{40}$. Los programas basados únicamente en la abstinencia no han mostrado eficacia, pese a ello algunos países aún implementan este tipo de programas ${ }^{13}$ lo que representa la posibilidad de causar daño. Pese a diversas fuerzas de oposición, en ALC se ha impulsado la EIS desde hace más de medio siglo, mediante la implementación de programas, la generación de enfoques -la interculturalidad, gestión de la diversidad, el abordaje con familias y el trabajo en el campo de la discapacidad-, herramientas técnicas, pedagógicas y políticas.

- Programas de prevención de embarazos en adolescentes (PEA): diversos países de ALC han adoptado planes y estrategias intersectoriales de PEA como respuesta a la alta tasa de fecundidad en adolescentes. Diversos países de la región han adoptado programas de PEA con diversos niveles de eficacia, por ejemplo, Uruguay ha implementado leyes y políticas progresistas, fuertes respuestas gubernamentales a nivel multisectorial de la mano con la participación activa de la sociedad civil, lo cual ha conducido a descensos en la fertilidad en adolescentes. Este abordaje multisectorial ha incluido los programas Uruguay Crece Contigo, espacios de educación y cuidados para hijas e hijos de estudiantes, servicios de salud sexual y reproductiva y el acceso a educación integral en sexualidad fuera de la escuela. El país ha obtenido resultados positivos, sin embargo, la implementación de programas ha encontrado desafíos ante la objeción de conciencia de proveedores de salud, así como disparidades en la formación docente en EIS que impactan la ampliación a escala ${ }^{41}$. La inversión en políticas de PEA debe observar la evidencia de OMS sobre las intervenciones eficaces para "Prevenir el embarazo precoz y los resultados reproductivos adversos en adolescentes en los países en desarrollo" ${ }^{\text {"42. }}$

- Sistemas de salud que respondan a las necesidades de adolescentes y jóvenes: en el marco de la Cobertura Universal de Salud estos programas buscan responder a las necesidades específicas y los derechos humanos de las juventudes. En ALC se han implementado servicios amigables para las personas jóvenes en países como Argentina, Colombia, Chile, El Salvador, Ecuador, Guatemala, México, Panamá y Uruguay, adhiriéndose en su mayoría a las Normas Mundiales para mejorar la calidad de los servicios de atención de salud de los adolescentes de OMS. Por ejemplo, en Chile, según Páez (2018 apud Venkatraman et al., 2019 ${ }^{43}$ ) a través de la implementación del Programa Salud Integral de Adolescentes y Jóvenes durante el periodo 2000-2017 lograron una reducción del $51 \%$ en la proporción de nacimientos en madres menores de 19 años. El programa fue llevado a escala a través de sistemas existentes, el desarrollo de capacidades y la regionalización gradual, garantizando su sostenibilidad mediante el uso de datos, la incidencia y diálogo político, permitiendo sostener el programa durante tres períodos de gobiernos con distintas posiciones ideológicas ${ }^{43}$.

- Programas centrados en niñas adolescentes (PCNA): la evidencia ha demostrado que, a través de la construcción de activos protectores -sociales, de salud, humanos, cognitivos y económicos- los programas de empoderamiento para niñas adolescentes (10-14 años) permitían mejorar considerablemente la inscripción y permanencia en la escuela, los conocimientos sobre sexualidad y uso de anticonceptivos, y aumentaban la edad del matrimonio ${ }^{44}$. Los PCNA buscan alcanzar de manera dirigida a las niñas adolescentes en condiciones de mayor vulnerabilidad; indígenas, afrodescendientes, rurales, pobres, que no viven con sus padres, migrantes, casadas o madres, trabajadoras domésticas, etc. Los PCNA cuando son abordados desde el modelo ecológico pueden mejorar resultados en educación, salud, economía, capital social, actitudes de género más equitativas y sobre la violencia, además aumentan su eficacia cuando se acompañan de transferencias de efectivo o en especie. En ALC se han implementado PCNA focalizados a nivel comunitario que han demostrado eficacia, por ejemplo, el Programa Abriendo Oportunidades en Guatemala que ha demostrado retrasar la edad del matrimonio y disminuir la violencia física y el Programa Abriendo Futuros en México ha demostrado mejorar el conocimiento en SSR y elevar la edad deseada para casarse o unirse ${ }^{45}$.

- Programas de masculinidades con hombres jóvenes: la revisión de evidencias señala que la estrategias con hombres y niños que han demostrado ser eficaces a nivel individual y comunitario para cambiar las actitudes y comportamientos de género incluyen una combinación de educación entre pares, utilizando defensores o mode- 
los masculinos, programas en medios de comunicación a gran escala, programas en el lugar de trabajo y programas comunitarios basados en derechos, cuyo objetivo es reducir la desigualdad entre los géneros y cambiar las normas sociales ${ }^{46}$. En ALC se han generado las bases de los programas para trabajar con jóvenes, el Programa $\mathrm{H}$ diseñado por una alianza de organizaciones civiles, se basa en el trabajo grupal participativo alrededor de más de setenta actividades orientadas por un currículo que aborda cuestiones de género, sexualidad y salud reproductiva, paternidad y cuidado, prevención de violencia y del Virus de Inmunodeficiencia Humana (VIH). Ocho evaluaciones, en su mayoría cuasi experimentales, han demostrado efectos positivos en actitudes y comportamientos de género más equitativos, mejoras en la comunicación de la pareja, reducción de la violencia basada en género y mejores actitudes en torno al cuidado ${ }^{47}$.

- Programas de prevención de violencia juvenil: con base en la perspectiva de juventud se destaca en este artículo la evidencia de intervenciones orientadas a cambios transformadores que tomen en cuenta el sistema patriarcal, desigual, discriminatorio y excluyente en el que se gesta y perpetúa la violencia, añadido a la necesidad de abordarla desde una visión democrática, sistémica y respetuosa de los derechos humanos ${ }^{48}$. Una de las prácticas eficaces es la regulación de la venta y consumo de alcohol, se ha demostrado que regular horarios de venta y consumo de alcohol en bares y restaurantes por las noches pueden reducir la violencia. Intervenciones promisorias para prevenir la violencia juvenil incluyen: a) la capacitación vocacional y el empleo que genera oportunidades y previene que las juventudes se involucren en violencia o actos delictivos, b) los programas de justicia restaurativa que generan procesos de mediación -entre las víctimas, victimarios, las familias y el sistema de justicia- los que incluyen orientación familiar son los que muestran los resultados más prometedores, c) las medidas no privativas de libertad que generan alternativas distintas al encarcelamiento ${ }^{49}$. La experiencia de las Escuelas Abiertas surgida en Brasil demostró resultados prometedores al ser implementada abriendo las escuelas durante los fines de semana y días festivos, cuyas evaluaciones observaron descenso de las principales expresiones de violencia, desarrollo de un mayor sentido de pertenencia, aumento de la autoestima personal y comunitaria y rendimiento escolar. Esta intervención ha sido replicada en Argentina, Guatemala, México y Uruguay ${ }^{50}$.

\section{Aún quedan caminos abiertos por recorrer}

ALC tiene una larga experiencia en la implementación de programas y políticas innovadoras con enfoques y miradas críticas que buscan resolver los problemas estructurales. Algunas intervenciones aplican el modelo ecológico, la integralidad y estrategias multicomponente que abordan los derechos humanos, el género y la racialidad. No obstante, los países enfrentan desafíos de inversión pública, laicidad, democratización, inclusión y protección social, sostenibilidad y expansión a escala de intervenciones dirigidas para las juventudes, lo cual permita alcanzar un triple dividendo que sostenga cambios generacionales.

En ALC iniciativas como la Declaración Ministerial Prevenir con Educación que convocó a los sectores de salud y educación en torno a la EIS y el acceso a servicios de SSR para las juventudes, así como mecanismos de observación, participación, incidencia política y auditoría social como Mira que te Miro en torno a los DSR resultan ser indispensables para la participación juvenil como parte del ejercicio de la ciudadanía, la exigibilidad de derechos y el avance democrático en la región.

Alcanzar la salud como el completo estado de bienestar para las juventudes en ALC, libres de violencias y opresión, donde sus derechos humanos sean garantizados, incluyendo los DSR, requiere asegurar que los sistemas y servicios públicos respondan a sus necesidades, sean entregados a través de múltiples plataformas intersectoriales, fortaleciendo la participación juvenil y los mecanismos de rendición de cuentas. Sólo así, los cambios políticos e institucionales alcanzados por la demanda y acción de la ciudadanía podrán ser sostenidos en el tiempo y contribuirán a que cada persona joven pueda decidir sobre su cuerpo, su vida y su mundo con dignidad y derechos ${ }^{51}$. 


\section{Referencias}

1. United Nations Population Fund (UNFPA). My Body, My Life, My World Rights and choices for all adolescents and youth: a UNFPA global strategy. New York: UNFPA; 2019.

2. Fondo de Población de las Naciones Unidas (UNFPA). 165 Millones de Razones, Un llamado a la acción para la inversión en adolescencia y juventud en América Latina y el Caribe. Panamá: UNFPA; 2018.

3. Comisión Económica para América Latina y el Caribe (CEPAL). Panorama Social de América Latina, 2019. Santiago: CEPAL; 2019.

4. Organización Panamericana de la Salud (OPS). La salud de los adolescentes y jóvenes en la Región de las Américas: la aplicación de la Estrategia y el Plan de acción regionales sobre la salud de los adolescentes y jóvenes (2010-2018). Washington, D.C: OPS; 2018.

5. Banco Mundial. Latinoamérica Indígena en el Siglo XXI. Washington, D.C: Banco Mundial; 2015.

6. Fondo de Población de las Naciones Unidas (UNFPA). Implicaciones del COVID-19 en la población afrodescendiente de América Latina y el Caribe. Panamá: UNFPA; 2019.

7. Organización Internacional del Trabajo (OIT). Panorama Laboral 2019 América Latina y el Caribe. Lima: OIT; 2019.

8. De Hoyos RE, Rogers H, Székely M. Ninis en América Latina: 20 millones de jóvenes en busca de oportunidades. Washington, D.C.: Banco Mundial; 2016.

9. Comisión Económica para América Latina y el Caribe (CEPAL). Los cuidados en América Latina y el Caribe. Textos seleccionados 2007-2018. Chile: CEPAL; 2018.

10. Organización Internacional de las Migraciones (OIM). Informe sobre las Migraciones en el Mundo 2018. Ginebra: OIM; 2018.

11. Gómez GM, Astaiza GM, Souza MC. Las migraciones forzadas por la violencia: el caso de Colombia. Cien Saude Colet 2008; 13(5):1649-1660.

12. Comisión Interamericana de Derechos Humanos (CIDH). Resolución 2/18 Migración forzada de personas venezolanas. Washington, D.C.: CIDH-OEA; 2018.

13. Organización Panamericana de la Salud (OPS). Fondo de Población de las Naciones Unidas (UNFPA). Fondo de las Naciones Unidas para la Infancia (UNI$\mathrm{CEF).} \mathrm{Acelerar} \mathrm{el} \mathrm{progreso} \mathrm{hacia} \mathrm{la} \mathrm{reducción} \mathrm{del} \mathrm{emba-}$ razo en la adolescencia en América Latina y el Caribe. Washington, D.C.: OPS; 2018.

14. The World Bank Group. Data. Adolescent fertility rate (births per 1,000 women ages 15-19). Washington, D.C: The World Bank; 2021.

15. Liang M, Simelane S, Fortuny G, Chalasani S, Weny K, Salazar P, Jenkins L, Moller AB, Venkatraman CM, Say L, Michielsen K, Engel DMC, Snow R. The State of Adolescents Sexual and Reproductive Health. J Adolesc Health 2019; 65:S3eS15.

16. Bongaarts J, Mensch BS, Blanc AK. Trends in the age at reproductive transitions in the developing world: The role of education. Pop Studies 2017; 71(2):139154.
17. Fondo de las Naciones Unidas para la Infancia (UNICEF). Perfil del matrimonio infantil y las uniones tempranas en América Latina y el Caribe. Nueva York: UNICEF; 2019.

18. Girls Not Brides. Matrimonio Infantil en América Latina y el Caribe. Londres: GNB; 2017.

19. Organización Mundial de la Salud (OMS). Informe sobre la situación mundial de la prevención de la violencia 2014.Resumen de Orientación. Ginebra: OMS; 2014.

20. Organización Panamericana de la Salud (OPS). Violencia juvenil interpersonal en América Latina y el Caribe de habla inglesa. Washington, D.C.: OPS-OMS; 2015.

21. World Health Organization (WHO). Global and regional estimates of violence against women: prevalence and health effects of intimate partner violence and non -partner sexual violence. Geneva: WHO; 2013.

22. Comisión Económica para América Latina y el Caribe (CEPAL). Indicadores femicidio. Chile: CEPAL-Observatorio de Igualdad de Género de América Latina y el Caribe; 2018.

23. OXFAM International. Informe Rompiendo moldes: transformar imaginarios y normas sociales para eliminar la violencia contra las mujeres. Reino Unido: OXFAM International; 2018.

24. Comisión Interamericana de Derechos Humanos (CIDH). Violencia contra personas Lesbianas, Gay, Bisexuales, Trans e Intersex en América. Washington, D.C.: OEA-CIDH; 2015.

25. Organización Panamericana de la Salud (OPS) Violencia juvenil autoinfligida en América Latina y el Caribe de habla inglesa. Washington, D.C.: OPS-OMS; 2015.

26. Banco Interamericano de Desarrollo (BID). Somos todos: Inclusión de las personas con discapacidad en América Latina y el Caribe. Washington, D.C.: IDB; 2019.

27. Luana M, Ortiz D, Urban AM. Violencia contra las mujeres y niñas con discapacidad: América Latina y el Caribe. Washington, D.C.: IDB; 2019.

28. Naciones Unidas. América Latina, no la más pobre pero sí la más desigual [Internet]. 2018. Disponible en: https://news.un.org/es/story/2018/04/1431712.

29. United Nations. Vienna Declaration and Programme of Action. Geneva: OHCHR; 2020.

30. United Nations. International Conference on Population and Development Programme of Action. New York: UNFPA; 2014

31. Naciones Unidas (NU). Declaración y Plataforma de Acción de Beijing. New York: UN Women; 2014.

32. Caroline W. Kabiru. Adolescents' Sexual and Reproductive Health and Rights: What Has Been Achieved in the 25 Years Since the 1994 International Conference on Population and Development and What Remains to Be Done? J Adolesc Health 2019; 65:S1eS2. 
33. Patton GC, Sawyer SM, Santelli, Ross DA, Afi fi R, Allen NB, Arora M, Azzopardi P, Baldwin W, Bonell C, Kakuma R, Kennedy E, Mahon J, McGovern T, Mokdad AH, Patel V, Petroni S, Reavley N, Taiwo K, Waldfogel J, Wickremarathne D, Barroso C, Bhutta Z, Fatusi AO, Mattoo A, Diers J, Fang J, Ferguson J, Ssewamala F, Viner RM. Our future: a Lancet commission on adolescent health and wellbeing. Lancet 2016; 387:2423-2478.

34. Hillenbrand E, Karim N, Mohanraj P, Wu D. Measuring gender-transformative change A review of literature and promising practices. Atlanta: CARE USA; 2015.

35. Starrs AM, Ezeh AC, Barker G, Basu A, Bertrand JT, Blum R, Coll-Seck A, Grover A, Laski L, Roa M, Sathar ZA, Say L, Serour GI, Singh S, Stenberg K, Temmerman M, Biddlecom A, Popinchalk A, Summers C, Ashford LS. Accelerate progress-sexual and reproductive health and rights for all: report of the Guttmacher-Lancet Commission. Lancet 2018; 391:2642-2692.

36. Naciones Unidas (NU). Estrategia Mundial para la Salud de la Mujer, el Niño y el Adolescente (2016-2030) Sobrevivir, prosperar, transformar. Nueva York: NU; 2015.

37. World Health Organization (WHO). Global Accelerated Action for the Health of Adolescents (AA-HA!) Guidance to Support Country Implementation. Geneva: WHO; 2017.

38. Organización Mundial de la Salud (OMS). Prevención de la Violencia: la evidencia. Serie de orientaciones sobre prevención de la violencia. Texas: OPS; 2013.

39. Aguilar L. Política Pública: Una visión panorámica. Bolivia: PNUD; 2012.

40. Naciones Unidas (NU). Orientaciones Técnicas Internacionales sobre educación en sexualidad. París: UNESCO; 2018.

41. Organización Mundial de la Salud (OMS). Directrices de la OMS para la prevención del embarazo precoz y los resultados reproductivos adversos en adolescentes de los países en desarrollo. Ginebra: OMS; 2011.

42. Venkatraman CM, Ferguson J, Plesons M, Paul M, Chalasani S, Amin A, Pallitto C, Sommers M, Avila R, Biaukula KVE, Husain S, Janušonyt $\mathrm{E}$, Mukherji A, Nergiz AI, Phaladi G, Porter C, Sauvarin J, Camacho AV, Engel DMC. The Political, Research, Programmatic, and Social Responses to Adolescent Sexual and Reproductive Health and Rights in the 25 Years Since the International Conference on Population and Development. J Adolesc Health 2019; 65(6 Suppl.):S16-S40.

43. Venkatraman CM, Plesons M, Hadley A, Maddaleno M, Oljira L, Tibebu S, Akwara E, Engel D. Lessons learned from national government-led efforts to reduce adolescent pregnancy in Chile, England and Ethiopia. Scaling Early Childhood Matters 2019; 2019:50-56.

44. Erulkar A, Muthengi E. Evaluation of Berhane Hewan: A Program to Delay Child Marriage in Rural Ethiopia. Int Perspect Sexual Reproductive Health 2009; 35(1):614.
45. Population Council. Delivering Impact for Adolescent Girls. Emerging Findings from Population Council Research. New York: PC; 2018.

46. Edström J, Hassink A, Shahrokh T, Stern E. Engendering Men: A Collaborative Review of Evidence on Men and Boys in Social Change and Gender Equality, EMERGE Evidence Review. Washington, D.C.: Promundo-US, Sonke Gender Justice and the Institute of Development Studies; 2015.

47. Promundo. Program H. Washington, D.C.: Promundo; 2020.

48. Aguilar I. Prevención de las violencias en el Triángulo Norte de Centroamérica. Desafíos Actuales. Rev Análisis Realidad Nacional 2020; 9(183):98-130.

49. Abdul Latif Jameel Poverty Action Lab (J-PAL) LAC. ¿Qué funciona para prevenir y reducir la violencia juvenil? Revisión sistemática de evidencias sobre prevención y reducción de la violencia juvenil con un análisis aplicado al contexto mexicano. República Dominicana: J-PAL; 2018.

50. Rodríguez E. Escuelas Abiertas, prevención de la violencia y fomento de la cohesión social en América Latina: experiencias destacadas y desafíos a encarar. Panamá: Parlatino-GIZ Prevenir; 2011.

51. Engel DMC, Paul M, Chalasani S, Gonsalves L, Ross DA, Venkatraman CM, Cole CB, Carvalho C, Hayes B, Philipose A, Beadle S, Ferguson BJ. A Package of Sexual and Reproductive Health and Rights Interventions - What Does It Mean for Adolescents? J Adolesc Health 2019; 65:S41eS50.

Artículo presentado en $16 / 06 / 2020$

Aprobado en 31/03/2021

Versión final presentada en 02/04/2021

Editores Jefes: Romeu Gomes, Antônio Augusto Moura da Silva 
